\title{
Weight optimization of variable weight OCDMA for triple-play services
}

\begin{abstract}
Triple-play services including video, voice and data demand service differentiation to provide end-users quality-of-service (QoS) requirements. Optical Code Division Multiple Access (OCMDA) systems have emerged as suitable solution to provide such QoS differentiation by varying the code weight. In this paper, the effect of choosing different code weight sets on the system capacity of a Variable-Weight OCDMA (VW-OCDMA) system deploying different detection techniques is investigated. Mathematical analysis is used to drive the Bit-Error Rate (BER) of active users in the system.
\end{abstract}

